ESAIM: PROCEEDINGS AND SURVEYS, November 2014, Vol. 46, p. 1-13

ECIT 2012 - Witold Jarczyk, Daniele Fournier-Prunaret, João Manuel Gonçalves Cabral

\title{
EXAMPLES ON PERRON EFFECTS IN AUTONOMOUS DISCRETE SYSTEMS
}

\author{
Francisco Balibrea and M.V. Caballero ${ }^{1}$
}

\begin{abstract}
We construct examples proving that there exist $C^{1}$-dynamical systems in $[0,1)$ having forward orbits instable in the sense of Lyapunov with negative Lyapunov exponent and forward orbits stable in the same sense, but having positive Lyapunov exponents. Such examples complete some results from Demir and Koçak for one dimensional systems and prove the appearance of Perron effects in such systems. Additionally we consider similar problems in two dimensional dynamical systems.

RÉSUMÉ. Nous construisons quelques examples pour démontrer qu'il y a de $C^{1}$-systèmes dinamiques dans $[0,1)$ avec la propiété d'avoir d'orbites devant instables au sens de Lyapunov avec des exposants négatifs au même sens, mais avec des exposants positifs de Lyapunov. Ces examples complétent des résultats de Demir et Koçak pour systèmes de dimension un et démontrent l'apparition des effets de Perron dans les systèmes. En plus de cela nous considérons des problèmes analogues dans des systèmes dynamiques de dimension deux.
\end{abstract}

\section{INTRODUCTION}

During years, a powerful tool to understand the behavior and predictability in nonlinear discrete dynamical systems and time series has been Lyapunov exponents. They have been used to decide when orbits are stable or instable in the Lyapunov sense. First it is necessary to remark that while stability in Lyapunov sense is a notion of topological character, Lyapunov exponents have a numerical nature and are calculated using the the derivative of maps in the points of orbits.

It is an extended practice, particularly in experimental dynamics, to associate having orbits with positive Lyapunov exponents with their instability and negative Lyapunov exponents with their stability. However we will see that such interpretations have no firm mathematical foundation if some restrictions on the maps describing dynamical systems are not introduced. In the rest of the paper, we will use only the terms of stability or instability, instead of using stability or instability in the sense of Lyapunov. In connection with the former statement, in [6] has been constructed two examples of dynamical systems defined by interval maps, one having an orbit with positive Lyapunov exponent but stable and other having one an orbit with negative Lyapunov exponent but instable. But such maps are highly non-differentiable and therefore we wonder if it is possible to obtain the same results but with differentiable maps. In section 4 we have obtained such examples in the semi-open interval $[0,1)$.

In fact the contributions of this paper are examples in discrete dynamical systems of the well known Perron effect. In [10] it is presented a survey and state of art of the problem mentioned in 1930 by Perron [12] who stated that in continuous dynamical systems, if the largest characteristic exponent of the first approximation

${ }^{1}$ Facultad de Matemáticas, Universidad de Murcia Campus de Espinardo, 30100 Murcia, Spain

(C) EDP Sciences, SMAI 2014 
ESAIM: PROCEEDINGS AND SURVEYS

of the system is negative, then the zero solution of the system could be Lyapunov instable and conversely. A discrete analog in the discrete case, was introduced by [10] and [7] in 2001. In fact Perron effect means a sign inversion of Lyapunov exponents of solutions of the original system to its associate system of its first approximation. Perron showed that negative largest Lyapunov exponent does not, in general, indicate stability and that positive largest Lyapunov exponents does not, in general, indicates instability. Moreover, in every neighborhood of the zero solution of the linear associate system, the original system can have solutions with positive characteristic exponents. Some other properties on regularity and irregularity are considered in [10].

In this paper we give more examples of the presence of Perron effect in two dimensional dynamical systems. Our construction could be extended to more dimensions. Such examples are constructed in the setting of autonomous systems, proving that even in such context the Perron effects can appear. By other hand we see that such effects appear in non-autonomous systems (see [1]). Our examples are less complicated than those introduced in [10].

In section 3 we prove a result in dynamical $C^{1}(I)$ with $I=[0,1]$ completing one stated in [6] relating the notion of strong Lyapunov exponents with stability or instability of orbits. In section 5 we construct two dimensional dynamical systems presented Perron effects. For them we will use the examples from section 4 . Before constructing them, it is necessary some introductory and preliminary definitions which are made in section 2

\section{Preliminary Definitions}

Given a dynamical systems $(X, f)$ where $X$ is a metric space and $f$ a continuous map from $X$ into itself, the forward orbit of a point $x \in X$ is the sequence $\left(f^{n}(x)\right)_{n=0}^{\infty}=\left(x_{n}\right)_{n=0}^{\infty}$ where as usual, $f^{n}$ denotes the $n$-iteration of $f$ acting on $x$. Two trajectories starting from nearby initial conditions can diverge or approach in some directions under $f$ when $n$ grows. Lyapunov exponents provide a means of quantifying the expansion or contraction on different directions of such trajectories.

Let $X \subset \mathbb{R}^{m}$ and $d$ any metric on it. If $\left(x_{n}\right)_{n=0}^{\infty}$ and $\left(x_{n}^{\prime}\right)_{n=0}^{\infty}$ are two trajectories starting from nearby initial states $x_{0}$ and $x_{0}^{\prime}$ and write $\delta x_{n}=x_{n}^{\prime}-x_{n}$. If $f$ has continuous partial derivatives in every $x_{i}$, then, iterating the map, we have the linear approximation, where $D f(x)$ denotes the differential of the map $f$ at the point $x$,

with the $(i, j)$ element of this matrix is given by $\frac{\partial f_{i}}{\partial x_{j}}$ and where $f_{i}$ and $x_{j}$ are the components of $f$ and $x$ in local coordinates on $X$ and

$$
D f^{n}\left(x_{0}\right)=D f\left(x_{n-1}\right) D f\left(x_{n-2}\right) \ldots D f\left(x_{1}\right) D f\left(x_{0}\right)
$$

where $\delta x_{n}$ represents the separation of theses orbits after $n$ iteration of the map $f$ which can be represented by the approximation

$$
\delta x_{n} \simeq D f^{n}\left(x_{0}\right) \delta x_{0}=\prod_{i=0}^{n-1} D f\left(x_{i}\right) \delta x_{0}
$$

The matrix $\left(D f^{n}\left(x_{0}\right)^{t}\right)\left(D f^{n}\left(x_{0}\right)\right)$ has $m$ eigenvalues given by $\mu_{i}\left(n, x_{0}\right)$ where $i=1,2, \ldots, m$ such that $\mu_{1}\left(n, x_{0}\right)|\geq| \mu_{2}\left(n, x_{0}\right)|\geq \ldots \geq| \mu_{m}\left(n, x_{0}\right) \mid$.

Definition 1. The ith local Lyapunov exponent at $x_{0}$ is defined by

$$
\lambda_{i}\left(x_{0}\right)=\lim _{n \rightarrow \infty} \frac{1}{2 n} \log \left(\left|\mu_{i}\left(n, x_{0}\right)\right|\right)
$$

if this limit exists.

In [11] was stated conditions for the existence of such limit. Now we recall the notions of instability and stability in the Lyapunov sense 
Definition 2. The forward orbit $\left(x_{n}\right)_{n=0}^{\infty}$ is sensitive to initial conditions or Lyapunov instable if there exists $\epsilon>0$ such that for any $\delta>0$ there exists $y$ with $d\left(x_{0}, y\right)<\delta$ and $N \geq 0$ such that $d\left(f^{N}(y), f^{N}\left(x_{0}\right)\right) \geq \epsilon$.

Definition 3. The forward orbit $\left(x_{n}\right)_{n=0}^{\infty}$ is not sensitive to initial conditions or it is Lyapunov stable if for any $\epsilon>0$ there is $\delta>0$ such that whenever $d\left(x_{0}, y\right)<\delta$ then $d\left(f^{n}(y), f^{n}\left(x_{0}\right)\right)<\epsilon$ for all $n \geq 0$.

When $n=1$, it easy to see that

Definition 4. The Lyapunov exponent $\lambda\left(x_{0}\right)$ of an orbit $\left\{x_{n}\right\}_{n=0}^{\infty}$ of the function $f$ is the number

$$
\lambda\left(x_{0}\right)=\lim _{n \rightarrow \infty} \frac{1}{n} \sum_{j=0}^{n-1} \log \left(\left|f^{\prime}\left(x_{j}\right)\right|\right)
$$

if this limit exists.

The definition of strong Lyapunov exponent was done in [8], where it is necessary the existence of the limit of definition 4 in a uniform way (definition 5). It is a sufficient condition to prove next theorem 1 (see [8]). We will use the same definition to obtain a similar result on stability.

Definition 5. The strong Lyapunov exponent $\Lambda\left(x_{0}\right)$ of a forward orbit $\left\{x_{n}\right\}_{n=0}^{\infty}$ is

$$
\Lambda\left(x_{0}\right)=\lim _{n \rightarrow \infty} \frac{1}{n} \sum_{j=k}^{k+n-1} \log \left(\left|f^{\prime}\left(x_{j}\right)\right|\right)
$$

if this limit exists uniformly with respect to $k \geq 0$

\section{Initial Perron examples}

In [12] in 1930, O.Perron introduced the following example for continuous dynamical systems

$$
\begin{gathered}
x_{1}^{\prime}(t)=-a x_{1}(t) \\
x_{2}^{\prime}(t)=(\sin (\log (t+1))+\cos (\log (t+1))-2 a) x_{2}(t)+x_{1}^{2}
\end{gathered}
$$

Later in [10] and [7] such examples where extended to the setting of the discrete system

$$
\begin{gathered}
x_{1}(n+1)=\exp ^{-a} x_{1}(n) \\
x_{2}(n+1)=\frac{\exp ((n+2) \sin \log (n+2)-2 a(n+1))}{\exp ((n+1) \sin \log (n+1)-2 a n)} x_{2}(n)+x_{1}(n)^{2}
\end{gathered}
$$

where $a$ is a number holding

$$
1<2 a<1+\frac{1}{2} \exp (-\pi)
$$

In both cases, in [10] it is referred that all Lyapunov exponents are negative, but almost all solutions of the original system tend exponentially to infinite. In such paper it is also proposed an example which show the possibility of sign reversal of Lyapunov exponents. In fact the Lyapunov exponent of the zero solution has a positive exponent while the solution of the original system having the same initial conditions has a negative exponent (see also [9]). 


\section{Results in Dimension one}

Theorem 1. Let $f \in C^{1}(I)$. If $\Lambda\left(x_{0}\right)>0$ for some $x_{0}$, then Orb $_{f}\left(x_{0}\right)$ is instable

Next result states that if an interval map is $C^{1}(I)$ and the orbit of $x_{0} \in I$ has a negative strong Lyapunov exponent, then such orbit is stable, which means that to assure stability of an orbit we need something more than the orbit to have a negative Lyapunov exponent if the map is only $C^{1}(I)$.

Theorem 2. Let $f \in C^{1}(I)$. If $\Lambda\left(x_{0}\right)<0$ for some $x_{0}$, then Orb $_{f}\left(x_{0}\right)$ is stable.

Proof. It is inspired in [8]. Write $a_{n}=f^{\prime}\left(x_{n}\right)$ (if $x_{n}=0$ or $x_{n}=1$ for some $n$, then it is understood we are using side-derivatives) and let $\left\{x_{n}\right\}_{n=0}^{\infty}=\operatorname{Orb}_{f}\left(x_{0}\right)$ and $\left\{y_{n}\right\}_{n=0}^{\infty}=\operatorname{Orb}_{f}\left(y_{0}\right)$ with $x_{0} \neq y_{0}$. Set $w_{n}=y_{n}-x_{n}$.

Let $w_{n+1}=a_{n} w_{n}+g_{n}\left(w_{n}\right)$ where

$$
g_{n}(w)=f\left(x_{n}+w\right)-f\left(x_{n}\right)-f^{\prime}\left(x_{n}\right) w
$$

with $\left|g_{n}(w)\right| \leq \omega(|w|)|w|$, where with $\omega($.$) we denote the modulus of continuity of the derivative. Then$

$$
g_{n}\left(w_{n}\right)=b_{n} w_{n}
$$

where

with

$$
b_{n}=\left\{\begin{array}{ccc}
\frac{g_{n}\left(w_{n}\right)}{w_{n}} & \text { if } & w_{n} \neq 0 \\
0 & \text { if } & w_{n}=0
\end{array}\right.
$$

$$
\left|b_{n}\right| \leq \omega\left(\left|w_{n}\right|\right)
$$

and $w_{n}$ can be considered as the solution of the lineal difference equation

$$
w_{n+1}=\left(a_{n}+b_{n}\right) w_{n}
$$

Using arguments from [8], page 384, we know that $\inf _{n \geq 0}\left|f^{\prime}\left(x_{n}\right)\right|=A \neq 0$

Suppose that $f$ has s.d.i.c. in $\operatorname{Orb}_{f}\left(x_{0}\right)$, that is, there exists $\epsilon>0$ such that for any $\delta>0$ there exists $y$ with $\left|y-x_{0}\right|<\delta$ and $N \geq 0$ such that

Let $N$ be the first index for which holds (1)

$$
\left|w_{N}\right|=\left|f^{N}(y)-f^{N}\left(x_{0}\right)\right| \geq \epsilon
$$

Initially choose $\epsilon$ such that:
i) $\Lambda\left(x_{0}\right)+\epsilon<\frac{\Lambda\left(x_{0}\right)}{2}<0$.
ii) $\omega(\epsilon)<A$ and $\frac{\omega(\epsilon)}{A} \leq\left|\frac{\Lambda\left(x_{0}\right)}{2}\right|$

According with the condition on strong Lyapunov exponent at $x_{0}$, for this $\epsilon$ there exists a positive integer $M$ such that for $n \geq M$ is

$$
\Lambda\left(x_{0}\right)-\epsilon<\frac{1}{n} \sum_{k=i+1}^{i+n} \log \left|a_{k}\right|<\Lambda\left(x_{0}\right)+\epsilon
$$

The proof is splitted in two parts. In the first is $N>M$ and in the second is $N \leq M$. Under such conditions we find two different contradictions, proving the statement. We consider two possibilities: 
- If $N>M$, as

$$
\left|w_{N}\right|=\left|a_{N-1}+b_{N-1}\right| \ldots\left|a_{N-M}+b_{N-M}\right|\left|w_{N-M}\right|
$$

with $\left|w_{N-M}\right|<\epsilon,\left|w_{N}\right| \geq \epsilon$ then

$$
\left|a_{N-1}+b_{N-1}\right| \ldots\left|a_{N-M}+b_{N-M}\right|>1 \Rightarrow \frac{1}{M} \sum_{j=1}^{M} \log \left|a_{N-j}+b_{N-j}\right|>0
$$

Knowing that $\log (1+x) \leq x$ we have

$$
\begin{aligned}
\frac{1}{M} \sum_{j=1}^{M} \log \left|a_{N-j}+b_{N-j}\right| & -\frac{1}{M} \sum_{j=1}^{M} \log \left|a_{N-j}\right|=\frac{1}{M} \sum_{j=1}^{M} \log \left|1+\frac{b_{N-j}}{a_{N-j}}\right| \leq \\
& \leq \frac{1}{M} \sum_{j=1}^{M}\left|\frac{b_{N-j}}{a_{N-j}}\right| \leq \frac{\omega(\epsilon)}{A} .
\end{aligned}
$$

Then by (2) and ii)

$$
\frac{1}{M} \sum_{j=1}^{M} \log \left|a_{N-j}+b_{N-j}\right| \leq \frac{1}{M} \sum_{j=1}^{M} \log \left|a_{N-j}\right|+\frac{\omega(\epsilon)}{A}<0 .
$$

This is a contradiction with (3)

- If $N \leq M$ we have

and by (1), $\sum_{k=1}^{N-1} \log \left|a_{k}+b_{k}\right|>0$.

$$
\left|w_{N}\right|=\left|a_{1}+b_{1}\right| \ldots\left|a_{N-1}+b_{N-1}\right|\left|w_{1}\right|
$$

Since

$$
\frac{1}{N-1} \sum_{k=1}^{N-1} \log \left|a_{k}+b_{k}\right|-\frac{1}{N-1} \sum_{k=1}^{N-1} \log \left|a_{k}\right|=\frac{1}{N-1} \sum_{k=1}^{N-1} \log \left|1+\frac{b_{k}}{a_{k}}\right| \leq \frac{\omega(\epsilon)}{A}
$$

we chose $\epsilon$ sufficiently small such that

$$
\frac{1}{N-1} \sum_{k=1}^{N-1} \log \left|a_{k}\right|>0
$$

Now by the definition of strong Lyapunov exponent and taken $n$ such that $n \geq M$, we have

$$
\Lambda\left(x_{0}\right)-\epsilon<\frac{1}{N+n-1}\left[\sum_{k=1}^{N-1} \log \left|a_{k}\right|+\sum_{k=N}^{N+n-1} \log \left|a_{k}\right|\right]<\Lambda\left(x_{0}\right)+\epsilon
$$

and

$$
\Lambda\left(x_{0}\right)-\epsilon<\frac{1}{n}\left[\sum_{k=N}^{N+n-1} \log \left|a_{k}\right|\right]<\Lambda\left(x_{0}\right)+\epsilon
$$

Taking the difference (5)-(6) we have

$$
-2 \epsilon<\frac{1}{N+n-1} \sum_{k=1}^{N-1} \log \left|a_{k}\right|+\left(\frac{1}{N+n-1}-\frac{1}{n}\right) \sum_{k=N}^{N+n-1} \log \left|a_{k}\right|<2 \epsilon
$$


that is,

$$
-2 \epsilon<\frac{1}{n+N-1} \sum_{k=1}^{N-1} \log \left|a_{k}\right|-\frac{N-1}{n(N+n-1)} \sum_{k=N}^{N+n-1} \log \left|a_{k}\right|<2 \epsilon
$$

Since this inequality holds for any $\epsilon$ sufficiently small, it must be

$$
\sum_{k=1}^{N-1} \log \left|a_{k}\right|-\frac{N-1}{n} \sum_{k=N}^{N+n-1} \log \left|a_{k}\right|=0
$$

that is,

$$
\sum_{k=1}^{N-1} \log \left|a_{k}\right|=\frac{N-1}{n} \sum_{k=N}^{N+n-1} \log \left|a_{k}\right|=0
$$

which is a contradiction since $\sum_{k=1}^{N-1} \log \left|a_{k}\right|$ is positive from (4) and $\frac{N-1}{n} \sum_{k=N}^{N+n-1} \log \left|a_{k}\right|$ is negative from (6).

\section{TWO NEW EXAMPLES IN DIMENSION ONE}

We construct two examples which are variants of the piecewise linear maps introduced in [6]. Our maps are $C^{1}([0,1))$ piecewise cubic maps. One of them has an orbit with negative strong Lyapunov exponent but instable and other with positive strong Lyapunov exponent but stable.

First step is to construct a cubic interval map $f(x)=a x^{3}+b x^{2}+c x+d$ such that $f\left(x_{1}\right)=y_{1}, f\left(x_{2}\right)=y_{2}$, $f^{\prime}\left(x_{1}\right)=t_{1}$ and $f^{\prime}\left(x_{2}\right)=t_{2}$ when $x_{1} \neq x_{2}$ because

$$
\left|\begin{array}{cccc}
x_{1}^{3} & x_{1}^{2} & x_{1} & 1 \\
x_{2}^{3} & x_{2}^{2} & x_{2} & 1 \\
3 x_{1}^{2} & 2 x_{1} & 1 & 0 \\
3 x_{2}^{2} & 2 x_{2} & 1 & 0
\end{array}\right| \neq 0
$$

where $a, b, c$ and $d$ can be calculated as:

$$
\begin{gathered}
a=\frac{2\left(y_{2}-y_{1}\right)-\left(t_{1}+t_{2}\right)\left(x_{2}-x_{1}\right)}{\left(x_{1}-x_{2}\right)^{3}} \\
b=\frac{t_{2}-t_{1}}{2\left(x_{2}-x_{1}\right)}-\frac{3}{2} a\left(x_{2}+x_{1}\right) \\
c=\frac{t_{1} x_{2}-t_{2} x_{1}}{x_{2}-x_{1}}+3 a x_{1} x_{2} \\
d=y_{1}+\frac{a}{2} x_{1}^{3}-\frac{3}{2} a x_{1}^{2} x_{2}+\frac{1}{2\left(x_{2}-x_{1}\right)}\left[\left(t_{2}+t_{1}\right) x_{1}^{2}-2 t_{1} x_{1} x_{2}\right]
\end{gathered}
$$

Example 1. We construct a continuous piecewise cubic map $f$ in I which is $C^{1}$ in $[0,1)$ and such that $\operatorname{Orb}_{f}(0)$ has a positive strong Lyapunov exponent and it is stable.

Such construction is made in several steps: 
a) Let us consider the points of $I$

$$
a_{n}<c_{n}<b_{n}<a_{n+1} \text { and } x \in I
$$

where $a_{n}=1-2^{-n}-10^{-n-1}, c_{n}=1-2^{-n}$ and $b_{n}=1-2^{-n}+10^{-n-1}$ for $n=0,1,2, \ldots$ and define the values of $f$ in such points by

$$
\begin{gathered}
f\left(a_{n}\right)=1-2^{-n-1}-2 \cdot 10^{-n-1} \\
f\left(c_{n}\right)=1-2^{-n-1} \\
f\left(b_{n}\right)=1-2^{-n-1}+2 \cdot 10^{-n-1} \\
f^{\prime}\left(a_{n}\right)=f^{\prime}\left(b_{n}\right)=0 \text { and } f^{\prime}\left(c_{n}\right)=2
\end{gathered}
$$

b) We denote by $f_{1}(x)$ the piece of cubic map joining the points $\left(a_{n}, f\left(a_{n}\right)\right)$ and $\left(c_{n}, f\left(c_{n}\right)\right)$ holding $f^{\prime}\left(a_{n}\right)=$ 0 and $f^{\prime}\left(c_{n}\right)=2$. This piece $f_{1}$ has the following coefficients

$$
\begin{gathered}
a=-2 \cdot 10^{2 n+2} \\
b=-2 \cdot 10^{n+1}+6 \cdot 10^{2 n+2}\left(1-2^{-n}\right) \\
c=2+4 \cdot 10^{n+1}\left(1-2^{-n}\right)-6 \cdot 10^{2 n+2}\left(1-2^{-n}\right)^{2} \\
d=1-2^{-n-1}-2 \cdot 10^{-n-1}+2 \cdot 10^{n+1}\left(1+10^{n+1}\left(1-2^{-n}\right)\right)\left(1-2^{-n}-10^{-n-1}\right)^{2}
\end{gathered}
$$

c) The piece of cubic map $f_{2}(x)$ joining $\left(c_{n}, f\left(c_{n}\right)\right),\left(b_{n}, f\left(b_{n}\right)\right)$ with $f^{\prime}\left(c_{n}\right)=2$ and $f^{\prime}\left(b_{n}\right)=0$ has the coefficients

$$
\begin{gathered}
a=-2 \cdot 10^{2 n+2} \\
b=2 \cdot 10^{n+1}\left(1+3 \cdot 10^{n+1}\left(1-2^{-n}\right)\right) \\
c=2-4 \cdot 10^{n+1}\left(1-2^{-n-1}\right)-6 \cdot 10^{2 n+2}\left(1-2^{-n}\right)^{2} \\
d=-1+3 \cdot 2^{-n-1}+2\left(1-2^{-n}\right)^{2} 10^{n+1}\left(1+\left(1-2^{-n}\right) 10^{n+1}\right)
\end{gathered}
$$

d) The coefficients of the piece of cubic map $f_{3}(x)$ joining $\left(b_{n}, f\left(b_{n}\right),\left(a_{n+1}, f\left(a_{n+1}\right)\right)\right.$ with $f^{\prime}\left(b_{n}\right)=0$ and $f^{\prime}\left(a_{n+1}\right)=0$ are

$$
\begin{gathered}
a=\frac{\left(10^{n+2}-2^{n+3} \cdot 11\right) 2^{2(n+1)} \cdot 10^{2(n+2)}}{\left(-10^{n+2}+11 \cdot 2^{n+1}\right)^{3}} \\
b=-\frac{3}{2} a\left(2-3 \cdot 2^{-n-1}+9 \cdot 10^{-n-2}\right) \\
c=3 a\left(1-2^{-n}+10^{-n-1}\right)\left(1-2^{-n-1}-10^{-n-2}\right) \\
d=1-2^{-n-1}+2 \cdot 10^{-n-1}+\frac{a}{2}\left(1-2^{-n}+10^{-n-1}\right)^{2}\left(-2+2^{-n-1}+13 \cdot 10^{-n-2}\right)
\end{gathered}
$$


Then the piecewise cubic map $f$ is defined by

$$
f(x)=\left\{\begin{array}{ccc}
f_{1}(x) & \text { if } & a_{n} \leq x \leq c_{n} \text { and } x \in I \\
f_{2}(x) & \text { if } & c_{n} \leq x \leq b_{n} \\
f_{3}(x) & \text { if } & b_{n} \leq x \leq a_{n+1} \\
1 & \text { if } & x=1
\end{array}\right.
$$

It is easy to test that $f$ is continuous at every $x \in I$ and $C^{1}$ in $[0,1)$. At each point of the orbit of 0 the derivative is 2 and therefore its strong Lyapunov exponent is $\lambda(0)=\log 2>0$, but this orbit is stable.

To see it, given $\epsilon>0$, we can chose $k$ such that $1 / 2^{k}<\epsilon$. Since $f$ is increasing and

$$
f^{k}(0)=1-\frac{1}{2^{k}} \text { then }\left|f^{n}(x)-f^{n}(0)\right| \leq \frac{1}{2^{n}}<\epsilon \text { for } n \geq k \text { and } 0<x<\bar{\delta}
$$

it remains to prove that the last inequality holds for $n<k$. It is made using that $f^{j}$ is continuous and then, given $\epsilon>0$ there exists $\delta_{j}$ such that if $0<x<\delta_{j},\left|f^{j}(x)-f^{j}(0)\right|<\epsilon$ for $j=1, \ldots, n-1$. Then if we take

$$
\delta=\min \left\{\delta_{1}, \ldots, \delta_{n-1}, \bar{\delta}\right\} \text { and } 0<x<\delta \Rightarrow\left|f^{k}(x)-f^{k}(0)\right|<\epsilon \text { for all } k>0
$$

Remark 1. This example does not contradict theorem 1 from [8] because our function is not $C^{1}$ in the compact interval $I$, but it is $C^{1}$ in $[0,1)$. It is crucial that the interval where the function is $C^{1}$ be non-compact.

Example 2. We construct a continuous piecewise cubic $C^{1}$-map $g$ in $[0,1)$ such that $\operatorname{Orb}_{g}(0)$ has a negative strong Lyapunov exponent and it is instable

a) First we consider the points of $I$ where the map connects the pieces:

$$
a_{n}<b_{n}<c_{n}<d_{n}<a_{n+1}
$$

where $a_{n}=1-2^{-n}-4^{-n-1}, b_{n}=1-2^{-n}, c_{n}=1-2^{-n}+4^{-n-1}$ and $d_{n}=1-2^{-n}+2 \cdot 4^{-n-1}$ for $n=1,2, \ldots$ and let $g$ map such that

$$
\begin{gathered}
g\left(a_{n}\right)=1-2^{-n-1}-(1 / 2) \cdot 4^{-n-1}, g\left(b_{n}\right)=1-2^{-n-1} \\
g\left(c_{n}\right)=1-2^{-n-1}+(1 / 2) \cdot 4^{-n-1}, g\left(d_{n}\right)=0 \\
g^{\prime}\left(a_{n}\right)=g^{\prime}\left(c_{n}\right)=g^{\prime}\left(d_{n}\right)=0 \text { and } g^{\prime}\left(b_{n}\right)=1 / 2
\end{gathered}
$$

b) The coefficients of piece cubic map $g_{1}(x)$ connecting $\left(a_{n}, g\left(a_{n}\right)\right)$ and $\left(b_{n}, g\left(b_{n}\right)\right)$ with $g^{\prime}\left(a_{n}\right)=0$ and $g^{\prime}\left(b_{n}\right)=1 / 2$ are

$$
\begin{gathered}
a=-2 \cdot 4^{2 n+1} \\
b=-2 \cdot 4^{n}+6 \cdot 4^{2 n+1}\left(1-2^{-n}\right) \\
c=4^{n+1}\left(1-2^{-n}\right)-6 \cdot 4^{2 n+1}\left(1-2^{-n}\right)^{2}+1 / 2 \\
d=1-2^{-n-1}-2 \cdot 4^{-n-2}+\left(1-2^{-n}-4^{-n-1}\right)^{2}\left(2 \cdot 4^{2 n+1}\left(1-2^{-n}\right)+2 \cdot 4^{n}\right)
\end{gathered}
$$


c) The piece of cubic map $g_{2}(x)$ connects $\left(b_{n}, g\left(b_{n}\right)\right)$ with $\left(c_{n}, g\left(c_{n}\right)\right)$ with $g^{\prime}\left(b_{n}\right)=1 / 2$ and $g^{\prime}\left(c_{n}\right)=0$ has the coefficients

$$
\begin{gathered}
a=-2 \cdot 4^{2 n+1} \\
b=2 \cdot 4^{n}+6 \cdot 4^{2 n+1}\left(1-2^{-n}\right) \\
c=(1 / 2)-4^{n+1}\left(1-2^{-n}\right)\left(1+6 \cdot 4^{n}\left(1-2^{-n}\right)\right) \\
d=(1 / 2)+2 \cdot 4^{n}\left(1-2^{-n}\right)^{2}\left(1+4^{n+1}\left(1-2^{-n}\right)\right.
\end{gathered}
$$

d) The piece of cubic map $g_{3}(x)$ which connects $\left(c_{n}, g\left(c_{n}\right)\right),\left(d_{n}, 0\right)$ with $g^{\prime}\left(c_{n}\right)=0$ and $g^{\prime}\left(d_{n}\right)=0$ has the coefficients

$$
\begin{gathered}
a=2 \cdot 4^{3(n+1)}\left(1-2^{-n-1}\right)+4^{2 n+2} \\
b=-\frac{3}{2} a\left(2\left(1-2^{-n}\right)+3 \cdot 4^{-n-1}\right) \\
c=3 a\left(1-2^{-n}+4^{-n-1}\right)\left(1-2^{-n}+2 \cdot 4^{-n-1}\right) \\
d=1-2^{-n-1}+2 \cdot 4^{-n-2}-\frac{a}{2}\left(1-2^{-n}+4^{-n-1}\right)^{2}\left(2\left(1-2^{-n}\right)+5 \cdot 4^{-n-1}\right)
\end{gathered}
$$

e) Finally, $g_{4}(x)$ is the piece of cubic map that connects $\left(d_{n}, 0\right),\left(a_{n+1}, g\left(a_{n+1}\right)\right)$ with $g^{\prime}\left(d_{n}\right)=g^{\prime}\left(a_{n+1}\right)=0$ and it has the following coefficients

$$
\begin{gathered}
a=\frac{-\left(2^{n+2} \cdot 4^{n+2}-4^{n+2}-2^{n+1}\right)\left(2^{2 n+4}\right)\left(4^{2 n+4}\right)}{\left(4^{n+2}-9 \cdot 2^{n+1}\right)^{3}} \\
b=\frac{-3 a}{2}\left(2-3 \cdot 2^{-n-1}+7 \cdot 4^{-n-2}\right) \\
c=3 a\left(1+2^{-n-1}-2^{-n}\right)\left(1-4^{-n-2}-2^{-n-1}\right) \\
d=\frac{a}{2}\left(1-2^{-n}+2 \cdot 4^{-n-1}\right)^{2}\left(-2+2^{-n-1}+11 \cdot 4^{-n-2}\right)
\end{gathered}
$$

The resultant cubic piecewise map is

$$
g(x)=\left\{\begin{array}{ccc}
g_{1}(x) & \text { if } & a_{n} \leq x \leq b_{n} \text { and } \\
g_{2}(x) & \text { if } & b_{n} \leq x \leq c_{n} \\
g_{3}(x) & \text { if } & c_{n} \leq x \leq d_{n} \\
g_{4}(x) & \text { if } & c_{n} \leq x \leq a_{n+1}
\end{array}\right.
$$

It is immediate that such map is $C^{1}$ in $[0,1)$. At each point of $\operatorname{Orb}_{g}(0)$, the derivative is $\frac{1}{2}$ and therefore its strong Lyapunov exponent is $\lambda(0)=-\log 2<0$.

Nevertheless, such orbit is instable. To see this, we can take $\epsilon=3 / 8$. For every $\delta>0$ there exists a $0<x<\delta$ and $k \geq 2$ such that $g^{k}(x)<1 / 2$ and $g^{k}(0) \geq 7 / 8$ and then

$$
\left|g^{k}(0)-g^{k}(x)\right|>\epsilon
$$

Remark 2. We underline again, that this example does not contradict our result of section 3, because this map is not $C^{1}$ in a compact interval, but it is $C^{1}$ in $[0,1)$. This it is a key point of the construction.

Remark 3. The pictures of the constructed maps are not shown in this paper because look like those presented in [6]. Our examples are the result of a rounding corners process of the cited piecewise linear examples from [6]

\section{TWO EXAMPLES IN DIMENSiÓN TWO}

We construct two dynamical systems, one defined in $[0,1]^{2}$ which has a forward orbit with a positive Lyapunov exponent but stable and other defined in $[0,1)^{2}$ which has a forward trajectory with a negative Lyapunov 
exponent but instable

Example 3. We construct a map $F=(f, g)$ in $[0,1]^{2}$ such that $\operatorname{Orb}_{F}(0,0)$ has a positive Lyapunov exponent, and it is stable.

a) The first component of $F$ is the map $f$ from [6]

$$
f(x)=\left\{\begin{array}{cc}
2 x-1+\frac{1}{2^{n+1}} & a_{n}<x \leq b_{n}, x \in[0,1] \\
\frac{5^{n+2}-22}{2 \cdot 5^{n+2}-11}\left(x-b_{n}\right)+1+\frac{2}{10^{n+1}}-\frac{1}{2^{n+1}} & b_{n}<x \leq a_{n+1} \\
1 & x=1
\end{array}\right.
$$

where $a_{n}=1-2^{-n}-10^{-n-1}, b_{n}=1-2^{-n}+10^{-n-1}, n=0,1,2, \ldots$

b) The second component of $F$ is $g:[0,1] \rightarrow[0,1]$ such that

$$
g(x)=\left\{\begin{array}{cc}
3 x+\frac{1}{2} & \leq x \leq \frac{1}{15} \\
\frac{6}{127} x+\frac{7}{10}-\frac{2}{635} & \frac{1}{15}<x \leq \frac{1}{2}-\frac{1}{100} \\
3 x+\frac{1}{2}-\frac{5}{2^{n+1}}\left(2^{n}-1\right) & a_{n}<x \leq b_{n} \\
\frac{5^{n+2}-33}{2 \cdot 5^{n+2}-11}\left(x-b_{n}\right)+1+\frac{3}{10^{n+1}}-\frac{1}{2^{n+1}} & b_{n}<x \leq a_{n+1} \\
1 & x=1
\end{array}\right.
$$

where $a_{n}=1-2^{-n}-10^{-n-1}, b_{n}=1-2^{-n}+10^{-n-1}, n=1,2, \ldots$

This map $F(x, y)=(f(y), g(x))$ is continuous in $[0,1]^{2}$, because $f$ y $g$ are continuous in $[0,1]$. We consider the trajectory of $(0,0)$ :

$$
\left\{(0,0),\left(x_{1}, y_{1}\right),\left(x_{2}, y_{2}\right), \cdots\right\}=\left\{\left(1-\frac{1}{2^{k}}, 1-\frac{1}{2^{k}}\right)\right\}_{k=0}^{\infty}
$$

In every points of this trajectory, the map is differentiable (except $(0,0)$ ). Since $f$ y $g$ are differentiable maps on right of 0 , we define

and

$$
D F\left(0^{+}, 0^{+}\right)=\left(\begin{array}{cc}
0 & \lim _{y \rightarrow 0^{+}} f(y) \\
\lim _{x \rightarrow 0^{+}} g(x) & 0
\end{array}\right)=\left(\begin{array}{ll}
0 & 2 \\
3 & 0
\end{array}\right)
$$

for $n=1,2, \ldots$.

$$
D F^{2 n}(0,0)=\left(\begin{array}{cc}
6^{n} & 0 \\
0 & 6^{n}
\end{array}\right) \quad D F^{2 n-1}(0,0)=\left(\begin{array}{cc}
0 & 2 \cdot 6^{n-1} \\
3 \cdot 6^{n-1} & 0
\end{array}\right)
$$

Now we compute the eigenvalues of $\left(D F^{k}\right)^{t}\left(D F^{k}\right)$ where $k=2 n$ or $k=2 n-1$ with $n=1,2, \ldots$. When $k=2 n-1$ :

$$
\left(D F^{2 n-1}(0,0)\right)^{t} D F^{2 n-1}(0,0)=\left(\begin{array}{cc}
3^{2} \cdot 6^{2(n-1)} & 0 \\
0 & 2^{2} \cdot 6^{2(n-1)}
\end{array}\right)
$$


and the largest eigenvalue of such matrix is $\mu_{1}(2 n-1,(0,0))=3^{2} 6^{n}$. When $k=2 n$ :

$$
\left(D F^{2 n}(0,0)\right)^{2}=\left(\begin{array}{cc}
6^{2 n} & 0 \\
0 & 6^{2 n}
\end{array}\right)
$$

whose the largest eigenvalue is $\mu_{1}(2 n,(0,0))=6^{2 n}$.

Therefore the Lyapunov exponent of the forward orbit with initial condition $(0,0)$ is

$$
\lambda_{1}(0,0)=\lim _{k \rightarrow \infty} \frac{1}{2 k} \log \left(\left|\mu_{1}(k,(0,0))\right|\right)=\log 6>0
$$

It is not difficult to prove that the forward trajectory of $(0,0)$ is stable. To see it, let $\epsilon>0$, we can chose $k>0$ such that $1 / 2^{k}<\epsilon$. As $f^{\prime}$ and $g^{\prime}$ are positive on the components of forward orbit of $(0,0)$, with the maximum distance that it is denoted by $|\cdot|$, we have that

$$
\left|F^{k}(x, y)-F^{k}(0,0)\right| \leq\left|\left(\frac{1}{2^{k}}, \frac{1}{2^{k}}\right)\right|=\frac{1}{2^{k}}<\epsilon
$$

for $n \geq k$ and $0<|(x, y)|<\bar{\delta}$. It remains to prove that the last inequality holds for $n<k$, but it is made using that $F^{j}$ is continuous and then, given $\epsilon>0$ there exists $\delta_{j}$ such that if $0<|(x, y)|<\delta_{j},\left|F^{j}(x, y)-F^{j}(0,0)\right|<\epsilon$ for $j=1, \ldots, n-1$. Then if we take

$$
\delta=\min \left\{\delta_{1}, \ldots, \delta_{n-1}, \bar{\delta}\right\} \text { and } 0<|(x, y)|<\delta
$$

it is true that

$$
\left|F^{k}(x, y)-F^{k}(0,0)\right|<\epsilon
$$

for all $k>0$.

Example 4. We construct a continuous function $G=\left(f^{2}, g\right)$ in $[0,1)^{2}$ such that $\operatorname{Orb}_{G}(0,0)$ has a negative Lyapunov exponent, and it instable.

a) The first component of $G$ is $f^{2}$ where the map $f:[0,1) \rightarrow[0,1)$ is defined in $[6]$ by

$$
f(x)=\left\{\begin{array}{cc}
\frac{1}{2} x+\frac{1}{2} & 0 \leq x<7 / 16 \text { or } a_{n} \leq x<b_{n} \\
\left(2^{n+1}-4^{n+1}-2^{-1}\right)\left(x+2^{-n}-2 \cdot 4^{-n-1}-1\right) & b_{n} \leq x<c_{n} \\
\frac{1-2^{-n-2}-2 \cdot 4^{-n-3}}{2^{-n-1}-9 \cdot 4^{-n-2}}\left(x+2^{-n}-2 \cdot 4^{-n-1}-1\right) & c_{n} \leq x<a_{n+1}
\end{array}\right.
$$

where $a_{n}=1-2^{-n}-4^{-n-1}, b_{n}=1-2^{-n}+4^{-n-1}, c_{n}=1-2^{-n}+2 \cdot 4^{-n-1}$ for $n=1,2, \ldots$

b) Now, the second component is $g:[0,1) \rightarrow[0,1)$ defined by 


$$
g(x)=\left\{\begin{array}{ccrl}
3 x+\frac{1}{2} & 0 \leq x \leq \frac{1}{15} \\
\frac{6}{127} x+\frac{7}{10}-\frac{2}{635} & \frac{1}{15}<y \leq \frac{1}{2}-\frac{1}{100} \\
3 x+\frac{1}{2}-\frac{5}{2^{n+1}}\left(2^{n}-1\right) & a_{n}<x \leq b_{n} \\
\frac{5^{n+2}-33}{2 \cdot 5^{n+2}-11}\left(x-b_{n}\right)+1+\frac{3}{10^{n+1}}-\frac{1}{2^{n+1}} & b_{n}<x \leq a_{n+1}
\end{array}\right.
$$

where $a_{n}=1-2^{-n}-10^{-n-1}, b_{n}=1-2^{-n}+10^{-n-1}$, para $n=1,2, \ldots$

The map $G(x, y)=\left(f^{2}(y), g(x)\right)$, is continuous in $[0,1)^{2}$ since $f$ and $g$ are continuous in $[0,1)$. Let us consider the trajectory of $(0,0)$, denoted by

$$
\left\{(0,0),\left(x_{1}, y_{1}\right),\left(x_{2}, y_{2}\right), \cdots\right\}
$$

where

$$
\left(x_{2 n}, y_{2 n}\right)=\left(1-\frac{1}{2^{3 n}}, 1-\frac{1}{2^{3 n}}\right) \quad \text { and } \quad\left(x_{2 n-1}, y_{2 n-1}\right)=\left(1-\frac{1}{2^{3 n-1}}, 1-\frac{1}{2^{3 n-2}}\right)
$$

for $n=1,2, \ldots$

Similarly to the former example, we have

$$
D G(0,0)=\left(\begin{array}{cc}
0 & 1 / 4 \\
3 & 0
\end{array}\right)
$$

and for $n=1,2, \ldots$

$$
D G^{2 n}(0,0)=\left(\begin{array}{cc}
(3 / 4)^{n} & 0 \\
0 & (3 / 4)^{n}
\end{array}\right) \quad D G^{2 n-1}(0,0)=\left(\begin{array}{cc}
0 & \frac{3^{n-1}}{4^{n}} \\
\frac{3^{n}}{4^{n-1}} & 0
\end{array}\right)
$$

Now we compute the largest eigenvalue of $\left(D G^{k}\right)^{t} \cdot D G^{k}$ when $k=2 n$ or $k=2 n-1$ with $n=1,2 \ldots$ If $k=2 n-1$, we have

$$
\left(D G^{2 n-1}(0,0)\right)^{t} D G^{2 n-1}(0,0)=\left(\begin{array}{cc}
\frac{3^{2 n}}{4^{2(n-1)}} & 0 \\
0 & \frac{3^{2(n-1)}}{4^{2 n}}
\end{array}\right)
$$

and $\mu_{1}(2 n-1,(0,0))=\frac{3^{2 n}}{4^{2(n-1)}}$. is:

And if $k=2 n$, we have $\mu_{1}(2 n,(0,0))=\frac{3^{2 n}}{4^{2 n}}$. Then the largest Lyapunov exponent of the orbit through $(0,0)$

$$
\lambda_{1}(0,0)=\lim _{k \rightarrow \infty} \frac{1}{2 k} \log \left(\left|\mu_{1}(k,(0,0))\right|\right)=\log \left(\frac{3}{4}\right)<0
$$

It is immediate to prove that $\operatorname{Orb}_{G}(0,0)$ is instable. For this we use the maximum distance which is represented by $|\cdot|$. 
Taking $\epsilon=3 / 8$, then for every $\delta>0$ there exists $(x, y)$ such that $|(x, y)|<\delta$ and $k \geq 2$ it is hold that $f^{k}<1 / 2$ and by other hand $f^{k}(0)>7 / 8$. Therefore

$$
\left|G^{k}(0,0)-G^{k}(x, y)\right|>\epsilon
$$

Remark 4. In a current work in progress, using similar procedures, we are extending the constructions made on $[0,1]^{2}$ or $[0,1)^{2}$ to $I^{n}$ or $[0,1)^{n}$ for $n>2$, using the permutation maps mentioned in [2].

\section{REFERENCES}

[1] F.Balibrea and M.V. Caballero, Stability of orbits via Lyapunov exponents in autonomous and non-autonomous systems. Int.J.Bifurcation and Chaos. 91, Vol 23, No 1,(2013) 13501-13517

[2] F.Balibrea, T.Caraballo, P.Kloeden and J.Valero, Recent developments in Dynamical Systems: three perspectives, Int.J.Bifur.Chaos, Vol 20, No.9, (2010), 2591-2636.

[3] J.Cánovas and A.Linero, Periodic structure of alternating continuous interval maps, J.Diff.Eqs.Appl., Vol 12, (2006), 847-858

[4] J.Cushing and S.Henson, A periodically forced Beverton-Holt equation, J.Diff.Equ.Appl., Vol8, (2002), 1119-1120

[5] E.Camouzis and G.Ladas, Periodically forced Pielou's equation, J.Diff.Equ.Appl., Vol13, (2007), 114-119

[6] B. Demir and S. Koçak, A note on positive Lyapunov exponent and sensitive dependence on initial conditions. Chaos, Solitons and Fractals 12 (2001), 2119 - 2121.

[7] I.V.Gayschun, Systems with discrete time (in russian), Inst. Matemat. RAN Belarusi, Minsk

[8] H. Koçak and K. Palmer, Lyapunov Exponents and Sensitive Dependence. Journal of Dynamics and Differential Equations 22(3), (2010), 381-398.

[9] G.A. Leonov, A modification of Perron's counterexample. Diff. Eqs. 39, (2010), 1651-1652.

[10] G.A.Leonov and N.V.Kuznetsov, Time-varying linearization and the Perron effects, International Journal of Bifurcation and Chaos, 17, No.4 (2007), 1079-1107.

[11] I. Osedelets , A multiplicative ergodic theorem. Lyapunov characteristic numbers for dynamical systems, Trans.Moscow Math.Soc. 19, (1968), 197-221.

[12] O.Perron, Die Stabilitatsfrage bei differentialgleichungen, Mathematische Zeitscrift, 32, 702-728.

Remark 5. This work has been partially supported by Grant MTM2011-23221 and CGL2008-05688-C02-02 from Ministerio de Ciencia e Innovación (Spain), Project 08667/PI-08 Fundación Séneca de la Comunidad Autónoma de Murcia (Spain). 